\title{
Data Extraction and Visualization from Three-dimensional Drawings Made in Immersive Virtual Environments
}

\author{
Savickaite, S., McDonnell, N. \& Simmons, D.R.
}

\begin{abstract}
One approach to characterizing human perceptual organization is to distinguish global and local processing. In visual perception, global processing enables us to extract the 'gist' of the visual information and local processing helps us to perceive the details. Individual differences in these two types of visual processing have been found in conditions like autism and ADHD. The ReyOsterrieth Complex Figure (ROCF) test is commonly used to investigate differences between local and global processing. Whilst Virtual Reality (VR) has become more accessible, cheaper, and widely used in psychological research, no previous study has investigated local vs global perceptual differences using immersive technology. In this study, we investigated individual differences in local and global processing as a function of autistic and ADHD traits. The ROCF was presented in the virtual environment and a standard protocol for using the figure was followed. A novel method of quantitative data extraction was used, which will be described in this paper in greater detail. Whilst some performance differences were found between experimental conditions, no relationship was observed between these differences and participants' levels of autistic and ADHD traits. Limitations of the study and implications of the novel methodology are discussed.
\end{abstract}

Keywords: Virtual Reality, 3D data, visualization, psychology, complex drawing task

\section{Index Terms:}

\section{INTRODUCTION}

Our visual system integrates a range of information to construct a meaningful perception of the world. The ability to detect and interpret such emerging visual patterns is often called "perceptual organisation"[1]. Individual experiences are private and inaccessible to experimenters, but there are distinct processing styles which are more amenable to investigation. Global processing is the ability to extract the 'gist' of the visual information presented, whereas local processing focuses on the details in a visual image. We all combine local elements of perception to extract the overall meaning. However, how we use this information to understand the world around us varies. Individual differences in local and global processing between individuals have been observed in infants [2]. Gender [3], race [4] and mood [5] have also been found to influence our preferred processing style. These differences extend to clinical and other populations, with distinct preferences observed in Obsessive Compulsive Disorder [6], schizophrenia [7], eating disorders [8], autism [9] and ADHD[10].

Autism Spectrum Disorders (ASD) are a group of neurodevelopmental conditions characterised by difficulties with social interactions, sensory processing and repetitive behaviour [11]. ASD directly affects around $1 \%$ of the UK population [12] and although the level of difficulty might vary, it undoubtedly affects everyday life. Self-report accounts from autistic individuals clearly show that their perception of the world is different from that of non-autistics [13]. 'Sensory overload' is often discussed as one of the key aspects of autistic perception, where everyday sensory stimulation accumulates to a point where it becomes overwhelming [14]. 'Fragmented world' is another type of perception where autistic individuals report seeing the world in isolated objects rather than the coherent whole picture apparently perceived by non-autistics [15].

ADHD is a neurodevelopmental condition characterised by patterns of inattention, hyperactivity and impulsivity [11]. ADHD traits have also been observed in the general population [16]. Some of the features of ADHD have been found to be similar to ASD with 30-50\% of ASD cases meeting ADHD criteria [17]. DSM-5 [11] allows the diagnosis of both "disorders" on the basis that both conditions share similar hereditary causes [18] and executive dysfunction [19]. When ASD and ADHD co-occur they often interact. Antshel et al [20] reported that people with ADHD, who also have co-diagnosed ASD, have more severe ADHD symptoms. Individuals with ADHD have also been found to be more challenged by global tasks [21] thus following similar patterns to autistics.

The Rey-Osterrieth Complex figure (ROCF) was designed by Andre Rey [22] and systemised by Paul-Alexandre Osterrieth [23] and was initially used in studies of neuropsychological cases, such as stroke or brain damage assessment (Figure 1). When administering the task, the figure is first presented, and the participant is asked to copy it on a piece of paper. Different colours are often used at different time increments to identify which elements of the picture participants draw first. The second part of the test investigates participants' ability to recall the figure. The copy can be made immediately, after a short delay or both. There are many scoring systems for the ROCF task. In our study we will use the perceptual [23] and organisational [24] scoring systems.

In recent years, VR has been used in ASD and ADHD research focusing on social interaction training and intervention. Social skills training has been performed with both autistic children [25] and adults [26]. Ke and $\mathrm{Im}$ [27] constructed a virtual dolphinarium for autistic children and Smith et al [28] developed an interview training set-up for autistic adults. These are just few examples of VR helping autistic people experience a world they normally are cautious of. Social and crowded situations are often a trigger for anxiety attacks in autism [29]. With VR autistic individuals can experience realistic scenarios without having to leave the safety of the room. Anxiety and phobia VR therapy in autism has proven to be successful and is now available via the NHS [30]. 
The aim of the study is to assess the interaction between individual differences and drawing capabilities in Virtual Reality (VR). As part of this pilot/exploratory experiment we have identified novel methodology for three-dimensional data extraction and visualization, specifically, the three-dimensional coordinates of complex drawings completed in TiltBrush.

\section{Pilot experiment}

\subsection{Participants}

Data were collected from January to June 2019 at the VR lab at the School of Humanities (Philosophy), University of Glasgow. Participants were recruited via Facebook post or word of mouth. Demographic information is summarised in Table 1.

\begin{tabular}{|l|}
\hline $8(38.1 \%)$ male Mean age 28.5 years ( $\mathrm{SD}=7.4)$ \\
\hline $13(61.9 \%)$ female Mean age 25.3 years ( $\mathrm{SD}=6.2)$ \\
\hline English speakers - 52.4\% \\
\hline 13 out of $21(61.9 \%)$ some form of corrected vision \\
\hline $15(44.2 \%)$ had experienced VR prior to the experiment \\
\hline
\end{tabular}

Figure 1: Table 1. Participant information.

\subsection{Materials}

Participants were tested using an Nvidia GTX 1080 I Gaming PC (Windows 10) with HTC Vive Pro kit. The experiment was run through TiltBrush (Google) using SteamVR software (Steam).

Two questionnaires were selected to assess individual differences amongst the participants: The Autism Spectrum Quotient and Adult ADHD Self-Report Questionnaires v 1.1. The Autism Spectrum Quotient Questionnaire (AQ) [31] is a questionnaire devised to assess autistic traits in the general population. It comprises 50 items, 10 for each of 5 subscales: social skill, attention switching, attention to detail, communication and imagination [32]. The questionnaire uses a 4-point agreement scale: definitely disagree, slightly disagree, slightly agree, definitely agree. Autistic traits are scored as 1 and non-autistic traits as 0 . Scores of $25+$ are considered high and are often an indicator that the individual might have diagnosable ASD [33]. The AQ has been found to be a good tool for investigating the continuum of autistic expression in the general population. Internal consistency and test-retest reliability of the questionnaire are consistent throughout different demographics and cultures [34]. It accurately supports the three-factor structure of ASD [35], which overlaps with the two-factor structure of DSM-5

The Adult ADHD Self-Report Questionnaire v 1.1 is an 18-item questionnaire devised to assess ADHD traits [36]. It has a 5-point answer scale: Never, Rarely, Sometimes, Often, Very Often. The first 6 questions are key in determining if the individual has ADHD traits and has been found to be most predictive of the condition. The remaining 12 questions provide additional details on the severity of the traits. Answers of Sometimes, Often or Very Often for Questions 1-3 and answers of Often and Very Often for Questions 4-6 are rewarded 1 point each. The maximum score is 6 . The questionnaire has consistently shown high convergent validity, correlation of subscales and test-retest reliability throughout different demographics and cultures [37]

\subsection{Procedure}

The study was conducted in accordance with University of Glasgow and Economic and Social Research Council (ESRC) ethical guidelines. Participants were introduced to VR via theBlu (WEVR). This game puts observers into an underwater scene. Observers cannot interact with the environment and spend around 5 minutes adapting to the surroundings and the virtual world. Short breaks are recommended for every VR experience. Due to the immersive quality of the tool it can sometimes disorient participants, particularly if they have not experienced VR prior to the experiment.

Participants were also introduced to the TiltBrush (Google) environment where they familiarised themselves with the functions of the controllers before proceeding to the first stage of the experiment. Participants were asked to draw the ROCF (Figure 1) in the three-dimensional virtual environment. They could freely choose the environment they wished to draw in (e.g. space, blank colour, winter scene), as well as the colour and brush they wished to draw in. Once the ROCF image was presented to them, participants could move it anywhere they found fit. After completion of the Copy condition, the ROCF image was removed from the environment and participants were asked to draw it from memory immediately and again, after a short delay. The AQ questionnaire was filled in after the Immediate Recall condition, which resulted in 15 minutes break from the VR. The final task was to draw the ROCF from memory again. After the Delayed Recall condition participants were asked to fill in the ASRS questionnaire and were debriefed.

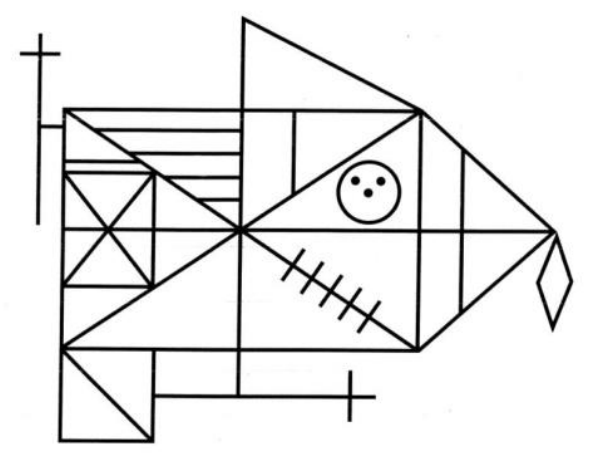

Figure 2: Figure 1. The Rey Osterrieth Complex Figure

\section{Data eXtraction}

TiltBrush data export contained all sketches as an .fbx file which had to be converted into an .obj file in order to obtain coordinates of the three-dimensional sketch. Conversion was completed using FBX Converter (Autodesk) and coordinates were obtained for all conditions. This has allowed a reconstruction of all sketches for statistical analysis. Converted .obj files could also be imported into MeshLab (GPL) for further analysis (Figure 2). The number of connected elements, depth of the sketch, surface area, perimeter and measurements of the boundary box were extracted. 


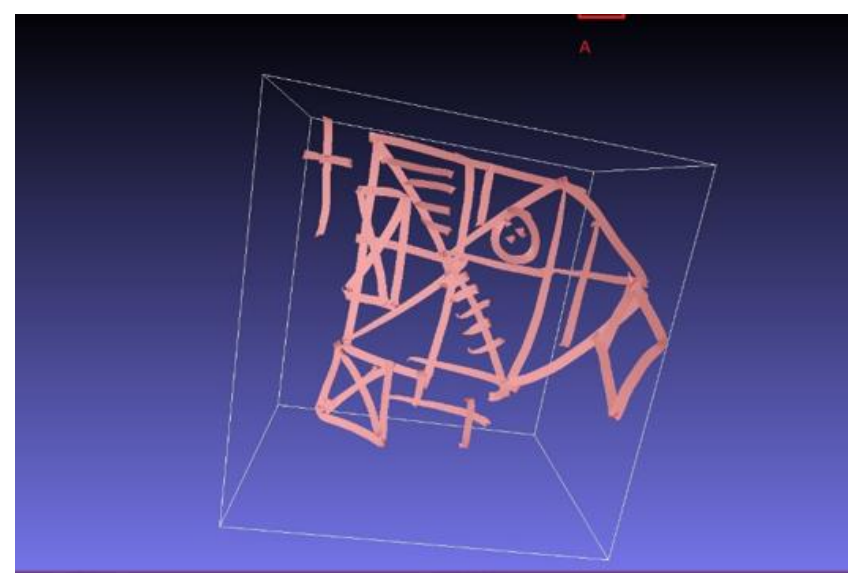

Figure 3: Figure 2. Example of 3D drawing recreated in MeshLab.

\section{Data visualisation}

3D Data were analysed. Coordinates were mapped and used for ROCF scoring and data visualization (Figure 3). As there were no time stamps attached to the coordinates, the sequence of the data points was divided into three equal parts to demonstrate which parts of the figure were drawn first (Figure 4).
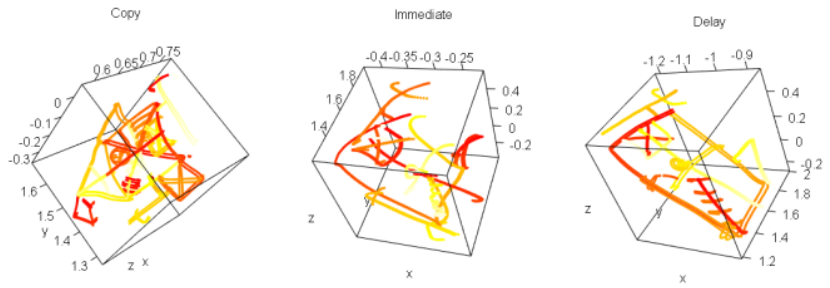

Figure 4: Figure 3. Coordinates mapped into 3D for one participant across the three experimental conditions.
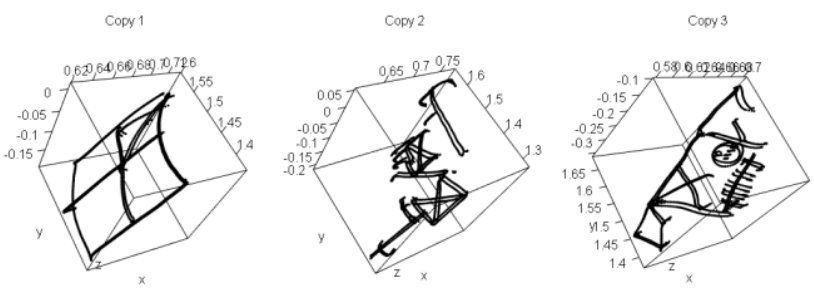

Figure 5: Figure 4. Coordinates of 3D sketch of a Copy condition has been divided into three parts to demonstrate how coordinates can be used to identify the temporal order of the elements drawn.

Videos were recorded for all three experimental conditions. Experimenters watched all the videos post experiment and recorded the type of brush and colours chosen by the participants and the number of deletions/adjustments of the drawing. In the Copy condition it was evident that participants were referring to the ROCF picture. The number of times an individual looked back at the reference image were counted. Videos were also used to record the total completion time for all three conditions.

\section{Results}

Participants all chose some form of marker to draw the figure. Some participants chose to use the same colour for all three conditions, whereas others changed colour for the Delayed Recall condition. The Percentage of colours chosen was calculated based on colours used by each participant (including any changes of colour between the conditions). Blue $(41.9 \%)$ was used most often, with Yellow/Orange (19.3\%), Pink (12.9\%) and Red $(12.9 \%)$ closely behind. Other colours used were White $(3.2 \%)$, Green $(3.2 \%)$ and Purple (6.4\%).

Completion times (min) differed significantly between the conditions $(\mathrm{F}(2,60)=6.3, \mathrm{p}<0.001)$. The Copy condition completion time (Mean $=3.2, \mathrm{SD}=1.8$ ) was significantly higher than the Immediate Recall condition (Mean $=2.1, \mathrm{SD}=0.9)$ and the Delayed Recall condition (Mean $=1.9, \mathrm{SD}=0.7$ ).

The number of times that participants referred to the ROCF picture in the Copy condition were calculated. The number of times looking at the figure was calculated and correlated with AQ scores $(r=-0.2, p=0.3)$ and ADHD scores $(r=0.1, p=0.7)$, but no significant correlations were found.

The number of deletions or alterations to the drawing were counted for each condition. The Delayed Recall condition (Mean $=3.2, \mathrm{SD}=4.5$ ) showed a slightly higher number of error corrections than the Copy (Mean $=2.5, \mathrm{SD}=3$.) and Immediate Recall (Mean $=1.5, \mathrm{SD}=1.7$ ) conditions. However, no significant differences were detected $(\mathrm{F}(2,60)=1.3, \mathrm{p}=0.3)$.

In order to calculate participants' completion rate, perceptual ROCF score was divided by the completion time (min). The completion rate for the Copy condition $($ Mean $=15.2, \mathrm{SD}=15.4)$ was higher than Immediate Recall (Mean $=13.7, \mathrm{SD}=9.2)$ and Delayed Recall $($ Mean $=12.8, \mathrm{SD}=4.8)$ conditions. Differences between the groups did not produce a significant difference $(\mathrm{F}(2$, $60)=0.3, p=0.8$ ). Due to high variability in the data high scorers (>40scores/min) were removed from the set. This has increased the $p$ value significantly $(F(2.54)=0.2, p=0.8)$. The low scorers $(<9$ scores/min) were removed for comparison. The $\mathrm{p}$ value decreased slightly but still did not reach significance $(\mathrm{F}(2.36)=$ $0.5, \mathrm{p}=0.6)$.
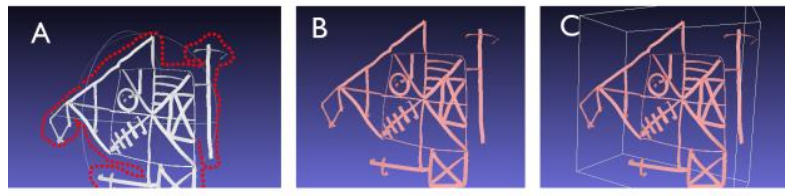

Figure 6: Figure 5. This Figure demonstrates data extracted from Meshlab: A - perimeter B - surface area (everything highlighted in pink); $\mathrm{C}$ - depth (extracted from the $\mathrm{x}, \mathrm{y}, \mathrm{z}$ axes coordinates for the boundary box).

There were no immediately observable differences between high and low scorers on the AQ. Thus, data extracted from MeshLab analysis was used to investigate the differences between conditions.

Only comparison of the number of Connected Elements between conditions showed significant difference $(F(2,60)=$ 
10.2, $\mathrm{p}<0.001)$. Perimeter $(\mathrm{F}(2,60)=0.1, \mathrm{p}=0.9)$, Surface Area $(\mathrm{F}(2,60)=0.2, \mathrm{p}=0.8)$ and Depth $(\mathrm{F}(2,60)=1.0, \mathrm{p}=0.4)$ did not show any significant differences between the three conditions (Figure 5A, 5B and 5C retrospectively).

\section{Discussion}

The aim of the study was to assess the interaction between individual differences and drawing capabilities in Virtual Reality (VR). As part of this pilot/exploratory experiment we have developed a novel methodology for the extraction and visualization of the three-dimensional coordinates of complex drawings completed using TiltBrush. Savickaite et al [38] collected additional data using the same experimental procedure and the questionnaires. The findings are available as a pre-print at https://psyarxiv.com/g7d9c/. The purpose of this paper is to discuss the methodology used in the 3D data extraction and visualization, and the potential for future work in this area.

Colour preferences add another level to the current investigation. The standard ROCF task often uses coloured markers to indicate different time intervals. In the current study these time intervals are generated automatically, and colour became a personal choice. Results indicated greater preferences for certain colours (blue and yellow/orange/red spectrum), but there was no obvious pattern to these choices, even though. atypical colour preferences have been reported in ASD and ADHD [39] [40]

Video data showed some differences in how many times participants looked at the figure during the copy condition. The ability to delete parts of the object in 3D is a new feature in the ROCF task. VR technology enabled us to extract this information. There were no significant differences between the numbers of deletions in the experimental conditions. The same patterns were observed in the 3D mesh data, including the number of connected elements, the perimeter, surface area and depth of the threedimensional figure.

The depth level of the sketches could have indicated differences between conditions, but no statistically significant differences were found. Depth precision in VR is poor and is an active field of research [41]. The depth information of the three-dimensional sketches might either indicate variable depth perception of the participants, or issues with the apparatus used. It can also suggest that both are responsible for the results observed, making it even harder to distinguish what is responsible for the differences observed. Perceptual differences in virtual reality should be explored separately in order to determine a suitable task for testing individual differences in processing styles.
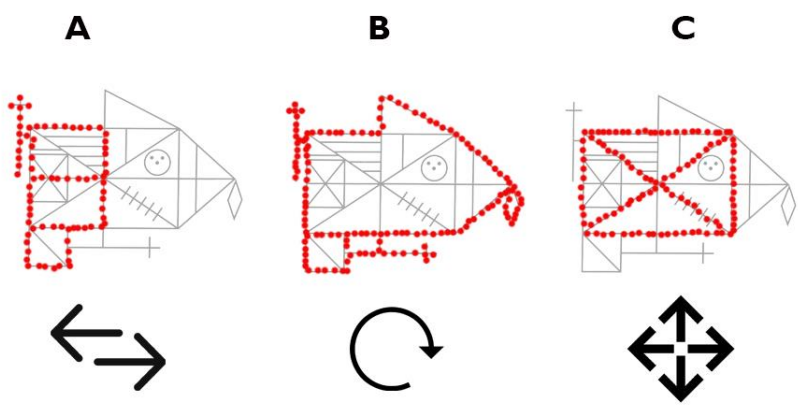

Figure 7: Figure 6. Strategies adopted by the participants were observed: A. drawing left to right or right to left; B. drawing the outline first and then filling in the details; C. following standardized global processing style, i.e. larger elements such as triangles and crosses are drawn first, and the details (such as small lines and the 'little face') filled in last.

Individual differences were evident in the video data and a more thorough qualitative analysis might reveal the distinct ways in which participants are approaching the task. Figure 6 illustrates just a few strategies adapted by the participants, where some prefer the standard global drawing style and others adopt alternative strategies of drawing (left to right or in a circular fashion). These alternative drawing styles are further discussed in Savickaite et al [38].

\section{REFERENCES}

[1] Wagemans, J., Elder, J. H., Kubovy, M., Palmer, S. E., Peterson, M. A., Singh, M., \& von der Heydt, R. (2012). A century of Gestalt psychology in visual perception: I. Perceptual grouping and figureground organization. Psychological bulletin, 138(6), 1172.

[2] Freeseman, L. J., Colombo, J., \& Coldren, J. T. (1993). Individual differences in infant visual attention: Four - month - olds' discrimination and generalization of global and local stimulus properties. Child Development, 64(4), 1191-1203.

[3] Roalf, D., Lowery, N., \& Turetsky, B. I. (2006). Behavioral and physiological findings of gender differences in global-local visual processing. Brain and cognition, 60(1), 32-42.

[4] McKone, E., Davies, A. A., Fernando, D., Aalders, R., Leung, H., Wickramariyaratne, T., \& Platow, M. J. (2010). Asia has the global advantage: Race and visual attention. Vision research, 50(16), 15401549.

[5] Gasper, K., \& Clore, G. L. (2002). Attending to the big picture: Mood and global versus local processing of visual information. Psychological science, 13(1), 34-40.

[6] Yovel, I., Revelle, W., \& Mineka, S. (2005). Who sees trees before forest? The obsessive-compulsive style of visual attention. Psychological science, 16(2), 123-129.

[7] Johnson, S. C., Lowery, N., Kohler, C., \& Turetsky, B. I. (2005). Global-local visual processing in schizophrenia: evidence for an early visual processing deficit. Biological psychiatry, 58(12), 937946.

[8] Lopez, C., Tchanturia, K., Stahl, D., \& Treasure, J. (2008). Central coherence in eating disorders: a systematic review. Psychological medicine, 38(10), 1393-1404.

[9] Kandalaft, M. R., Didehbani, N., Krawczyk, D. C., Allen, T. T., \& Chapman, S. B. (2013). Virtual reality social cognition training for young adults with high-functioning autism. Journal of autism and developmental disorders, 43(1), 34-44.

[10] Wang, M., \& Reid, D. (2011). Virtual reality in pediatric neurorehabilitation: attention deficit hyperactivity disorder, autism and cerebral palsy. Neuroepidemiology, 36(1), 2-18.

[11] American Psychiatric Association Division of Research. (2013). Highlights of changes from DSM-IV to DSM-5: Somatic symptom and related disorders. Focus, 11(4), 525-527.

[12] Baird, G., Simonoff, E., Pickles, A., Chandler, S., Loucas, T., Meldrum, D., \& Charman, T. (2006). Prevalence of disorders of the autism spectrum in a population cohort of children in South Thames: the Special Needs and Autism Project (SNAP). The lancet, 368(9531), 210-215.

[13] Grandin, T., \& Duffy, K. (2008). Developing talents: Careers for individuals with Asperger syndrome and high-functioning autism. AAPC Publishing. 
[14] Robertson, A. E., \& Simmons, D. R. (2015). The sensory experiences of adults with autism spectrum disorder: A qualitative analysis. Perception, 44(5), 569-586.

[15] Frith, U. (2003). Autism: Explaining the enigma. Blackwell Publishing.

[16] Lubke, G. H., Hudziak, J. J., Derks, E. M., van Bijsterveldt, T. C., \& Boomsma, D. I. (2009). Maternal ratings of attention problems in ADHD: evidence for the existence of a continuum. Journal of the American Academy of Child \& Adolescent Psychiatry, 48(11), 1085-1093.

[17] Panagiotidi, M., Overton, P. G., \& Stafford, T. (2018). The relationship between ADHD traits and sensory sensitivity in the general population. Comprehensive psychiatry, 80, 179-185.

[18] Lichtenstein, P., Carlström, E., Råstam, M., Gillberg, C., \& Anckarsäter, H. (2010). The genetics of autism spectrum disorders and related neuropsychiatric disorders in childhood. American Journal of Psychiatry, 167(11), 1357-1363.

[19] Rommelse, N. N., Geurts, H. M., Franke, B., Buitelaar, J. K., \& Hartman, C. A. (2011). A review on cognitive and brain endophenotypes that may be common in autism spectrum disorder and attention-deficit/hyperactivity disorder and facilitate the search for pleiotropic genes. Neuroscience \& Biobehavioral Reviews, 35(6), 1363-1396.

[20] Antshel, K. M., Hier, B. O., \& Barkley, R. A. (2014). Executive functioning theory and ADHD. In Handbook of executive functioning (pp. 107-120). Springer, New York, NY.

[21] Song, Y., \& Hakoda, Y. (2018). Selective impairment of basic emotion recognition in people with autism: discrimination thresholds for recognition of facial expressions of varying intensities. Journal of autism and developmental disorders, 48(6), 1886-1894.

[22] Rey, A. (1941). L'examen psychologique dans les cas d'encéphalopathie traumatique.(Les problems.). Archives de psychologie.

[23] Osterrieth, P. A. (1944). Le test de copie d'une figure complexe; contribution a l'etude de la perception et de la memoire. Archives de psychologie.

[24] Hamby, S. L., Wilkins, J. W., \& Barry, N. S. (1993). Organizational quality on the Rey-Osterrieth and Taylor Complex Figure Tests: A new scoring system. Psychological assessment, 5(1), 27.

[25] Matsentidou, S., \& Poullis, C. (2014, January). Immersive visualizations in a VR cave environment for the training and enhancement of social skills for children with autism. In 2014 International Conference on Computer Vision Theory and Applications (VISAPP) (Vol. 3, pp. 230-236). IEEE.

[26] Bellani, M., Fornasari, L., Chittaro, L., \& Brambilla, P. (2011). Virtual reality in autism: state of the art. Epidemiology and psychiatric sciences, 20(3), 235-238.

[27] Ke, F., \& Im, T. (2013). Virtual-reality-based social interaction training for children with high-functioning autism. The Journal of Educational Research, 106(6), 441-461.

[28] Smith, M. J., Ginger, E. J., Wright, K., Wright, M. A., Taylor, J. L., Humm, L. B., ... \& Fleming, M. F. (2014). Virtual reality job interview training in adults with autism spectrum disorder. Journal of autism and developmental disorders, 44(10), 2450-2463.

[29] Kuriakose, S., \& Lahiri, U. (2016). Design of a physiology-sensitive VR-based social communication platform for children with autism. IEEE Transactions on Neural Systems and Rehabilitation Engineering, 25(8), 1180-1191.

[30] Maskey, M., Lowry, J., Rodgers, J., McConachie, H., \& Parr, J. R. (2014). Reducing specific phobia/fear in young people with autism spectrum disorders (ASDs) through a virtual reality environment intervention. PloS one, 9(7), e100374.

[31] Baron-Cohen, S. (2011). Zero degrees of empathy: A new theory of human cruelty. Penguin uk.

[32] Baron-Cohen, S., Wheelwright, S., Hill, J., Raste, Y., \& Plumb, I. (2001). The "Reading the Mind in the Eyes" Test revised version: a study with normal adults, and adults with Asperger syndrome or high-functioning autism. The Journal of Child Psychology and Psychiatry and Allied Disciplines, 42(2), 241-251.

[33] Woodbury-Smith, M. R., Clare, I. C. H., Holland, A. J., \& Kearns, A. (2006). High functioning autistic spectrum disorders, offending and other law-breaking: findings from a community sample. The Journal of Forensic Psychiatry \& Psychology, 17(1), 108-120.

[34] Stevenson, J. L., \& Hart, K. R. (2017). Psychometric properties of the autism-spectrum quotient for assessing low and high levels of autistic traits in college students. Journal of Autism and Developmental Disorders, 47(6), 1838-1853.

[35] Hurst, R. M., Mitchell, J. T., Kimbrel, N. A., Kwapil, T. K., \& Nelson-Gray, R. O. (2007). Examination of the reliability and factor structure of the Autism Spectrum Quotient (AQ) in a non-clinical sample. Personality and Individual Differences, 43(7), 1938-1949.

[36] Kessler, R. C., Adler, L., Ames, M., Demler, O., Faraone, S., Hiripi, E. V. A., ... \& Walters, E. E. (2005). The World Health Organization Adult ADHD Self-Report Scale (ASRS): a short screening scale for use in the general population. Psychological medicine, 35(2), 245256.

[37] Silverstein, M. J., Alperin, S., Faraone, S. V., Kessler, R. C., \& Adler, L. A. (2018). Test-retest reliability of the adult ADHD SelfReport Scale (ASRS) v1. 1 Screener in non-ADHD controls from a primary care physician practice. Family practice, 35(3), 336-341.

[38] Savickaite, S., McNaughton, K., Gaillard, E., Amaya, I., McDonnell, N., Millington, E., \& Simmons, D. (2021). Using HMD Virtual Reality to investigate individual differences in visual processing styles.

[39] Grandgeorge, M., \& Masataka, N. (2016). Atypical color preference in children with autism spectrum disorder. Frontiers in psychology, 7, 1976.

[40] Hill, M. (2011). Young Children and Their Perceptions of Colour: An Exploratory Study.

[41] Zhao, H., \& Wu, B. (2020). Three-dimensional face modeling technology based on $5 \mathrm{G}$ virtual reality binocular stereo vision. International Journal of Communication Systems, e4651. 